\title{
Historische Darstellungen blühender Agaven
}

\author{
Hermann Maué \& Hilke Steinecke
}

\begin{abstract}
The American Century Plant (Agave americana) was known as Aloe americana in pre-Linnean times. The flowering of a Century Plant in noble gardens marked a major event. Flowering specimens were depicted in artwork and medals of various sorts. Here we present some prominent examples.
\end{abstract}

\section{Zusammenfassung}

Die Amerikanische Agave (Agave americana) war in vorlinnéscher Zeit unter dem Namen Aloe americana bekannt. Wenn Agaven in herrschaftlichen Gärten zur Blüte kamen, war das eine große Attraktion. Blühende Exemplare der sog. Aloe americana wurden auf Gemälden, Kupferstichen und Medaillen abgebildet. Einige Beispiele hierfür werden vorgestellt.

\section{Aloe americana - ein herrschaftliches Gewächs}

Die Amerikanische Agave wurde schon früh aus der Neuen Welt nach Europa gebracht. Aufgrund ihrer Ähnlichkeit mit Aloen (rosettig angeordnete, sukkulente Blätter) beschrieb sie Carolus Clusius als Aloe americana (Clusius 1576). Bekannt machte die so genannte Aloe americana eine großformatige und prächtige Abbildung im Hortus Eystettensis von 1613 (Besler 1613). Erst Carl v. Linné prägte den Namen Agave americana (v. Linné 1753). Aloen und Agaven gehören nach moderner Auffassung beide zu der Ordnung der Spargelartigen (Asparagales) und werden neuerdings wieder einer Familie zugeordnet. Aloen und Agaven werden zu den Grasbaumgewächsen (Xanthorrhoeaceae) gezählt.

Die bei uns nicht winterharten Amerikanischen Agaven wurden nördlich der Alpen zunächst nur in herrschaftlichen Gärten kultiviert, wo sie im Kübel gehalten wurden und den Winter in einem geheizten Raum, z. B. in einer Orangerie, verbrachten. Wenn derartige Agaven nach mehreren Jahrzehnten endlich zur Blüte kamen, war das ein ganz besonderes Ereignis, das ausführlich dokumentiert und auf verschiedene Art und Weise dargestellt wurde.

Abb. 1: Johann Georg Puscher, auf die Blüte einer Aloe in Nürnberg, Kupferstich, 1726.

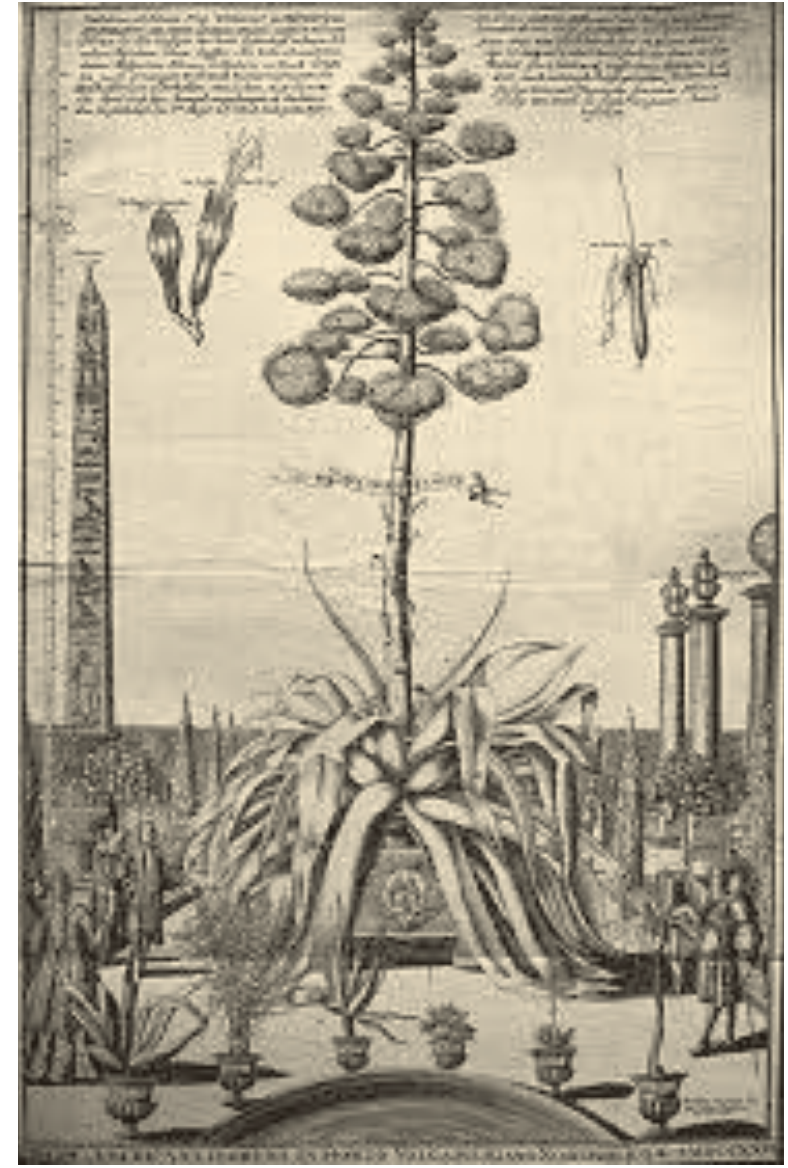

Viele historische Darstellungen blühender Agaven stammen aus vorlinnéscher Zeit, weshalb man hier noch die Bezeichnung Aloe americana findet (UllRICH 1993). Bis zum Jahre 1800 sind in Mitteleuropa 95 Blühereignisse dokumentiert. Die Blüte einer Agave interpretierte man als ein glückliches Vorzeichen, mit dem man Segenswünsche für das Fürstenhaus oder 
auch für die Vaterstadt verband. Darstellungen blühender Agaven wurden oft auf ganz bestimmte Personen bezogen. Sie regten zur Erfindung von Emblemen an, die auch in die Medaillenkunst Eingang fanden. Einige Beispiele historischer Agavendarstellungen werden exemplarisch vorgestellt.

\section{Agavendarstellungen auf Kupferstichen und in der Dichtung}

Blühende Agaven wurden vermessen, ihre Höhe sowie die Zahl ihrer Verzweigungen und Blüten genauestens festgehalten. Oft wurden sie dann auch auf Kupferstichen verewigt. So blühte z. B. im Jahr 1726 in Nürnberg im VolKamerschen Garten eine Amerikanische Agave. Johann Magnus Volkamer ließ nicht nur eine Medaille prägen, sondern bestellte beim Verleger Johann Georg Puscher gleich auch einen großformatigen Kupferstich mit der Darstellung dieser Agave und der Inschrift: „Diese Aloe ist nicht durch Kunst getrieben, sondern durch fleisige Warte und Pflegung des Gaertners Johann Georg Weidners, zu diesem Florisanten Stand befördert" (Abb. 1). Während die Volkamersche Agave den ganzen Sommer lang lediglich vor Wind und Regen geschützt unter freiem Himmel stand, wendeten andere Gärtner gewisse Tricks an, um die Blütenstände zu induzieren.

Manche Gärtner schworen darauf, die Agaven das ganze Jahr über in geheizten Räumen zu halten, um das Wachstum zu beschleunigen. Es wird berichtet, dass eine Agave, wenn man sie abgeschnitten unter die Decke eines Raumes hängt und die Wurzeln zwischendurch nicht anfeuchtet, ein Jahr und länger nicht nur grün bleibt und weiter wächst, sondern auch noch Blüten bildet. Heinrich Hesse hält in seinem „Teutschen Gärtner“von 1710 fest, man könne die „Aloe im Winter aus dem geschirr nehmen, [müsse] aber ein wollen tuch in trocken leinöl, so mit kreide vermenget, netzen, und solches um die wurtzel schlagen oder wickeln, und an einen warmen trockenen ort biß in den april aufhencken, da es dann wieder in seine vorgedachte erde kann gepflantzet werden" (Hesse 1710).
In der Tat scheint die erste Methode auch nach Erfahrung des Palmengartens relativ effektiv zu sein, um (Not)Blüten hervorzubringen. Vor einigen Jahren musste im Trockenwälder- und Savannenhaus des Tropicariums eine große Agaven-Verwandte (Furcraea) aus Platzgründen und wegen thematischer Umgestaltung des Schauhauses entfernt werden. Im Frühling wurden beim Ausgraben der Rosette die Wurzeln größtenteils zerstört. Die nahezu wurzellose, aber noch attraktive Blattrosette wurde noch während des Sommers in einem großen Kübel im Sommer-Sukkulentengarten gezeigt. Gegen Ende der Saison entwickelte sich ein mehrere Meter hoher Blütenstand aus der noch immer grünen Rosette. Die Furcraea wurde vor den ersten Frösten in das Schauhaus gestellt. Bis zum Jahresende hatten sich an dem Blütenstand mehrere hundert glockenförmige Blüten geöffnet. Danach trocknete die Rosette ein.

Auch in der Dichtkunst wird die blühende Agave gepriesen und gilt als gutes Omen und als Zeichen für späte Erfüllung lang gehegter Wünsche. In der 5. Strophe der 1713 verfassten „Trostaria“"von Johann Christian GÜNTHer (1696-1723) heißt es:

Endlich blüht die Aloe, Endlich trägt der Palmbaum Früchte, Endlich schwindet Furcht und Weh, Endlich wird der Schmerz zunichte, Endlich sieht man Gottes Tal: Endlich, endlich kommt einmal.

\section{Agaven als Emblem}

Agaven mit ihrer Symbolik wurden auch als Embleme verwendet. Die erste Medaille, auf der eine Agave als Emblem erschien, entstand 1625 auf den Tod des erst 15-jährigen Herzogs Georg Wilhelm von Liegnitz Brieg und Wohlau, der nach nur neunmonatiger vormundschaftlicher Regentschaft starb (Abb. 4). Auf dessen Tod schuf Samuel Koller eine Medaille mit dem Porträt des Herzogs auf der Vorderseite und einer blühenden Agave in einem Blumenkübel auf der Rückseite. Noch nicht alle Blüten haben sich geöffnet, doch fal- 


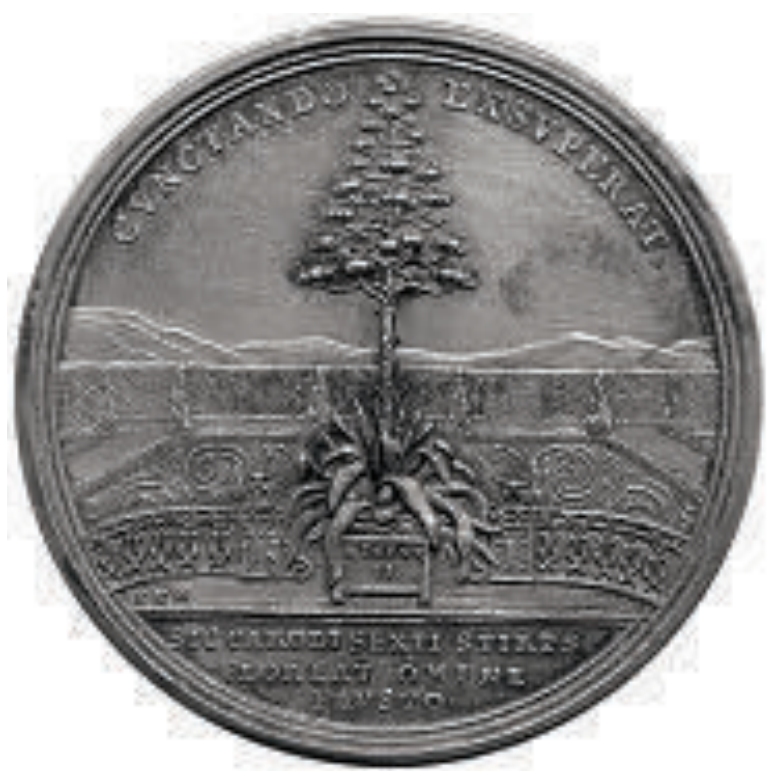

len bereits Fruchtkapseln aus der Blütenkrone herab. Die nach reicher Blüte absterbende Agave ist ein Sinnbild des frühen Todes des Herzogs. Die lateinische Umschrift lautet sinngemäß: Ich sterbe, sobald ich geblüht habe. Daniel Casper von Lohenstein greift dieses Motiv auf: „Er, der Hertzog, ist verdorret, wie die Aloe-Staude, welche nach Herfürbringung so viler köstlicher Blumen, also bald Safft und Leben verlieret." (Dewerdeck 1711).

\section{Agavenmedaillen}

Medaillen, die auf das Blühereignis ganz bestimmter Agaven geprägt wurden, dienten zudem als eine Art Protokoll, in dem die Fakten des Blühereignisses festgehalten werden sollten. Häufig sind aber auch Lebensweisheiten und Segenswünsche auf den Medaillen zu finden. Den frühesten Auftrag für eine Medaille auf die im Jahr 1700 erschienene Blüte einer Agave erteilte der Leipziger Kaufmann Caspar Bose (1672-1730) dem Medailleur Georg Wilhelm Kittel (Abb. 4). Sein so genannter Großbosischer Garten war besonders im 18. Jh. weit über die Grenzen Leipzigs hinaus bekannt. Auf der Vorderseite der Medaille ist eine Agave in ei-

Abb. 2: Peter Paul Werner, auf die Blüte einer Aloe in Nürnberg, 1726

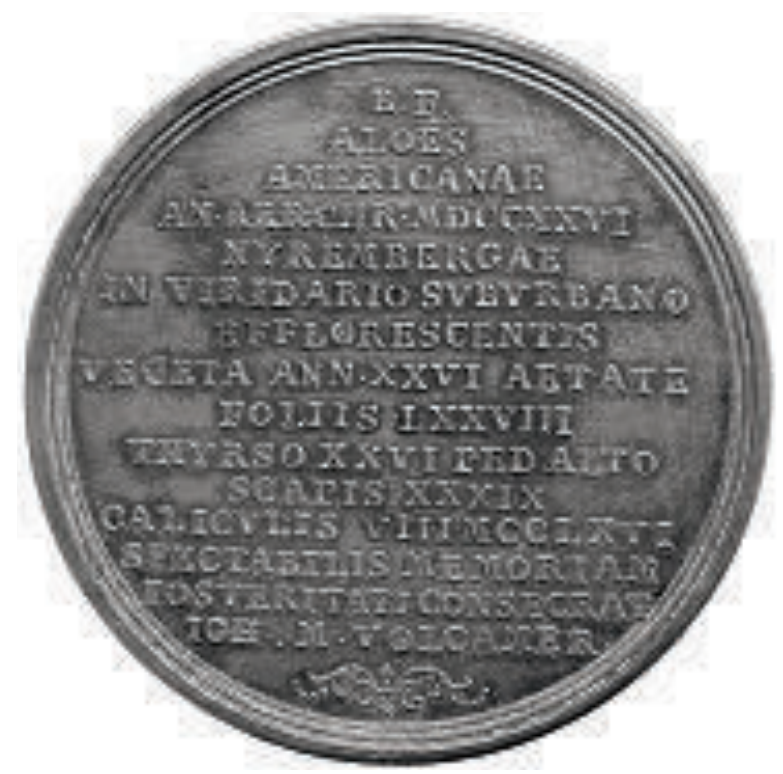

nem Blumenkübel dargestellt. Die lateinische Inschrift besagt: Der Bosische Garten in Leipzig sah, dass die Amerikanische Aloe im Alter von 28 Jahren bis zu einer Höhe von 24 Fuß getrieben hat mit 35 Zweigen und 5138 Blüten. Gewürdigt wurde auch der Gärtner (Cultor E. Pein). Auf der Rückseite ist Leipzig dargestellt. Seiner Stadt wünscht Bose ein langes Gedeihen nach dem Motto „So möge auch Leipzig fort und fort blühen". Aus botanischer Sicht passen die Blüte der Agave und ein langes Leben der Stadt eigentlich nicht zusammen, da die Agave bekanntlich unmittelbar nach der Blüte abstirbt.

Im Jahre $1710 \mathrm{kam}$ in Gotha eine Agave in einem Lustgarten von Herzog FrIedrich von Sachsen-Gotha-Altenburg zur Blüte (Abb. 5). Christian Wermuth zeigt auf einer Medaille diese Agave. Es handelt sich um ein Exemplar mit zehn niedrigen Schäften, ähnlich dem kürzlich in Bruchköbel erblühten und in diesem Heft beschriebenen Exemplar. Die zehn Schäfte sind mit den Namen der sechs damals lebenden und der vier verstorbenen Kinder des Herzogs bezeichnet. Auch hier wird der für die Agave verantwortliche Gärtner Georg BenjAMIN KunTZ gewürdigt. Auf der Rückseite wird in 27 Zeilen die Agave in lateinischer Sprache beschrieben, wobei es hier vor allem auf das gute Omen für den Besitzer ankommt. In Übersetzung bedeutet die Inschrift: 

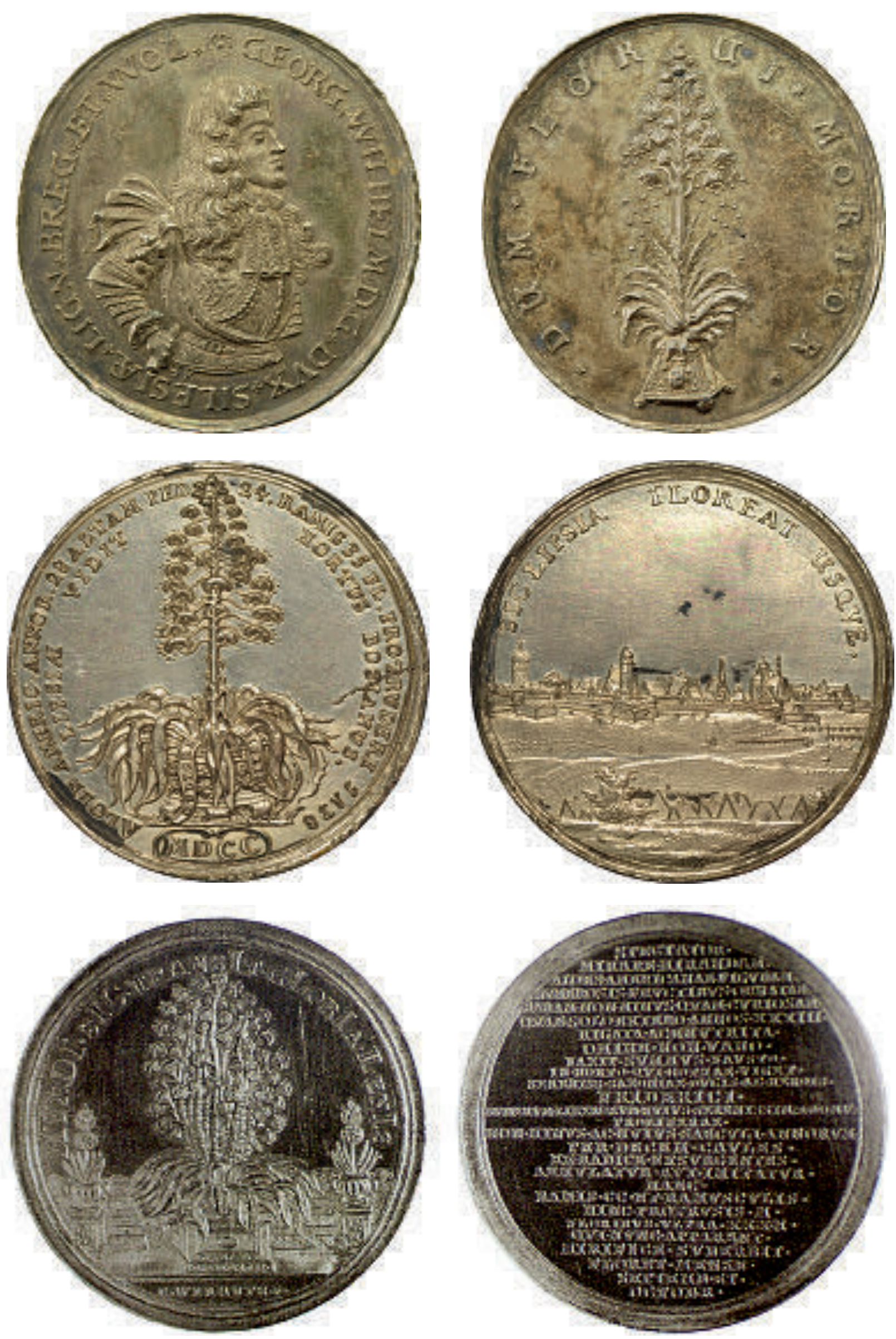
„Betrachter, bewundere das bewundernswerte, das seltene und nicht weniger bemerkenswerte Aussehen der mit unzähligen Früchten geschmückten amerikanischen Aloe, die 33 Jahre draußen im Garten nur gegossen und gedüngt wurde. Entsprechend diesem nicht bedeutungslosen Vorzeichen gebe der Höchste, dass in FrIEDRICHs, des durchlauchtigsten Herzogs von Sachsen und Helden, glücklichem Garten, der in Gotha blüht, die Zahl der Kinder des durchlauchtigsten, fruchtbaren Hauses nicht weniger sei, und der Zahl der Jahre dieses Jahrhunderts will diese es durch die zehn aus der Wurzel heraustreibenden Schäfte gleichtun oder ahmt sie sogar nach mit ihren zweihundert Ästen und tausend dort ausragenden Zweigen und mit den mehr als dreißigtausend Blüten, die nun auf wunderbare Weise erscheinen. Sie strotzt und blüht im Monat September und Oktober."

Eine mehrschäftige Agave war für die Botaniker der damaligen Zeit ein Rätsel. Angedacht, aber dann ausgeschlossen wurde die Vermutung, dass der Gärtner zehn einzelne Agaven in einen Blumentrog gepflanzt hat und alle im selben Jahr geblüht haben. Nur drei Jahre später heißt es aber in der „Europäischen Fama“, dass diese Agave „aus ihrem zerteilten Hertzen zehn gerade Stangen" trieb und mehr als dreißigtausend Blüten hervorbrachte (Die Europäische Fama 1713).

Mittlerweile sind uns blühende Agaven vertraut, denn sie sind in beliebten Urlaubsregionen wie im Mittelmeerraum oder auf den Kanaren schon lange verwildert und treten $z$. T. in Massenbeständen auf. Die Blüten- bzw. alten Fruchtstände prägen mancherorts das Landschaftsbild. Dennoch ist es immer noch faszinierend, historische Abbildungen blühender

Abb. 3 (oben): Samuel Koller, auf den Tod Herzog Georg Wilhelms von Liegnitz Brieg und Wohlau, 1675.

Abb. 4 (Mitte): Johann Kittel, auf die Blüte einer Aloe im Bosischen Garten zu Leipzig, 1700.

Abb. 5 (unten): Christian Wermuth, auf die Blüte einer Aloe im Schlossgarten von Friedrichsthal, Gotha, 1710.
Agaven zu betrachten oder bei uns z. B. in Botanischen Gärten verschiedenste Arten blühender Agaven anzuschauen. Nicht umsonst wählte LinNé den wissenschaftlichen Namen Agave aus, der aus dem Griechischen abzuleiten ist und so viel wie edel, prachtvoll oder erhaben bedeutet. Agaven sind königlich, denn ihr Name könnte sich auch auf die Agaue (andere Schreibweise: Agave) beziehen. Laut griechischer Mythologie ist Agaue eine Tochter von Kadmos (Sohn des phönizischen Königs Agenor von Tyros) und Harmonia (Göttin der Eintracht).

Königlich beschrieben wird auch eine 44-jährige Agave, die 1712 in Köpenick erblühte: „Die Aloe ist das fürnehmste Gewächs im Reich der Blumen, ihre Grösse, Schönheit, Alter, Tugend und Wachsthum, und übrige Vorrechte der Natur, geben ihr den Preiß, daß sie von aller Welt als die Königin unter den Blumen angesehen und bewundert wird. Sofern ist sie ein Sinnbild seiner Königl. Majest. in Preussen." (WACHTER 1712)

Ausführlichere Beschreibungen und weiterführende Literatur sind MaUÉ (2015) zu entnehmen.

\section{Literatur}

Besler, B. 1613: Hortus Eystettensis. - Nürnberg. Clusius, C. 1579: Aromatvm, et simplicivm aliqvot medicamentorvm apvd indios nascentivm historia. Antwerpen: $12-13$.

Dewerdeck, G. 1711: Silesia nunmismatica, oder Einleitung zu dem Schlesischen Müntz-Cabinet. Jauer: 399.

Die Europäische Fama 1713: Die Europäische Fama, Welche den gegenwärtigen Zustand der vornehmsten Höfe entdecket, Teil 145. - Leipzig: 558-561.

Hesse, H. 1710: Teutscher Gärtner. Das ist Eine gründliche Vorstellung, wie [...] ein Lust-Küchen-und Baum-Garten füglich anzurichten. - Leipzig: 105.

v. Linné, C. 1753: Species Plantarum. - Stockholm: 323-324.

Maué, H. 2015: Die Amerikanische Aloe, die Königin der Blumen. - Geldgeschichtliche Nachrichten 279/280: 236-250.

UlLRICH, B. 1993: Agaven. Illustrationen blühender Exemplare bis 1800. - Palmengarten Sonderheft 21. Frankfurt.

Wachter, J. G. 1712: Application der Cöpenickischen Aloe auf das königl. Preußische Haus in Heroischen Devisen. In: Theatrum Europaeum. 19: 1712. Frankfurt 1723: 213-215. 